\title{
USE OF 3D PROTOTYPES FOR COMPLEX SURGICAL ONCOLOGIC CASES
}

Short Title: 3D Printed Tumor Models

Keywords: Surgical Oncology, 3D Printing, Tumor Models

Word Count: 1780

Conflict of interest: None

Lucas Krauel MD (Corresponding Author)

Pediatric Surgery Department

Hospital Sant Joan de Déu, Universitat de Barcelona

Passeig de Sant Joan de Déu, 2

08950 Barcelona

SPAIN

Ikrauel@hsjdbcn.org

Phone: +34932532156

Fax: $\quad+34932532149$

Felip Fenollosa Engr.

Fundació CIM

Department of Mechanical Engineering

ETSEIB, Universitat Politècnica de Catalunya

Diagonal 647

08028 Barcelona

SPAIN

ffenollosa@fundaciocim.org 
Pediatric Radiology Department

Hospital Sant Joan de Déu, Universitat de Barcelona

Passeig de Sant Joan de Déu, 2

08950 Barcelona

SPAIN

Iriaza@hsjdbcn.org

Martin Perez Engr.

Fundació CIM

Department of Mechanical Engineering

ETSEIB, Universitat Politècnica de Catalunya

Diagonal 647

08028 Barcelona

SPAIN

martinpereztorrents@gmail.com

Xavier Tarrado MD PhD

Pediatric Surgery Department

Hospital Sant Joan de Déu, Universitat de Barcelona

Passeig de Sant Joan de Déu, 2

08950 Barcelona

SPAIN

xtarrado@hsjdbcn.org

\section{Andres Morales MD}

Pediatric Oncology Department

Hospital Sant Joan de Déu, Universitat de Barcelona

Passeig de Sant Joan de Déu, 2

08950 Barcelona

SPAIN

amora@hsjdbcn.org

Joan Gomà Engr. PhD 
Fundació CIM

Department of Mechanical Engineering

ETSEIB, Universitat Politècnica de Catalunya

Diagonal 647

08028 Barcelona

SPAIN

jgoma@fundaciocim.org

Jaume Mora MD

Pediatric Oncology Department

Hospital Sant Joan de Déu, Universitat de Barcelona

Passeig de Sant Joan de Déu, 2

08950 Barcelona

SPAIN

jmora@hsjdbcn.org

\section{ABSTRACT:}

Introduction:

Physical 3D models known by the industry as rapid prototyping involve the creation of a physical model from a 3D computer version. In recent years there has been an increasing number of reports on the use of 3D models in medicine. Printing such 3D models with different materials integrating the many components of human anatomy is technically challenging. In this article we report our technological developments along with our clinical implementation experience using high fidelity 3D prototypes of tumors encasing major vessels in anatomically sensitive areas.

Methods:

Three patients with tumors encasing major vessels that implied complex surgery were selected for surgical planning using 3D prototypes. 3D virtual models were obtained from routine CT and MRI images. The models, with all their anatomical relations, were created by an expert pediatric radiologist and a surgeon, image by image, along with a computerized aid designer (CAD) engineer.

Results: 
Surgeons had the opportunity to practice on the model before the surgery. This allowed questions regarding surgical approach; feasibility and potential complications to be raised in advance of the actual procedure.. All patients then successfully underwent surgery as planned.

Conclusion:

Having a tumor physically printed in its different main component parts with its anatomical relationships is technically feasible. Since a Gross Total Resection is prognostic in a significant percentage of tumor types, refinements in planning may help achieve greater and safer resections therefore contributing to improve surgical management of complex tumors. In this early experience, 3D prototyping helped significantly in the many aspects of surgical oncology planning.

\section{INTRODUCTION:}

Physical 3D models known by the industry as rapid prototyping involves the creation of a physical model from a 3D computer version. This technology started in 1987 when the first stereolithography machine was commercialized. In recent years there has been an increasing number of reports on the use of 3D models in medicine for teaching, diagnosis, surgical planning and bone reconstructions[1-5]. Using 3D printing for boney structures is very straightforward. Therefore most of the published literature in medicine is based on maxillofacial and orthopedic cases. However, fewer experiences are being described about soft tissue surgical planning 3D printing [6-9]. Printing 3D models with different materials integrating the many components of the anatomy is technically more challenging. Surgical oncology dealing with tumors encasing major vessels can be difficult to perform and careful planning is mandatory.

In this article we report our technological developments and clinical implementation experience gained from high fidelity 3D prototypes of tumors encasing major vessels in anatomically sensitive areas. The models were designed from patients routine computed tomography (CT) and Magnetic Resonance Image (MRI) imaging studies.

\section{METHODS:}

\section{Patients and Tumors}

Three patients with tumors encasing major vessels that implied complex surgery were selected for surgical planning using 3D prototypes before the operation. A summary of the patients and tumor characteristics are shown in table 1.

Case \#1 is a 3-year-old male with stage 4 MYCN amplified, high-risk, neuroblastoma (NB). After mN7 induction chemotherapy, the metastatic disease presented a complete response. Imaging studies showed a suprarenal tumor extending to the midline, encasing the right renal artery, the right renal vein, the inferior vena cava (IVC) and the superior mesenteric artery (SMA). Local control with surgery was indicated. 
Case \#2 is a 5-year-old male with a stage 4 MYCN amplified, high-risk, NB. After mN7 induction chemotherapy the metastatic disease was in complete remission. A midline-centered mass remained encasing the celiac trunk, SMA, inferior mesenteric artery (IMA), both renal arteries, the renal veins, IVC, portal vein, the right hepatic artery and the hepatic duct. Local control was advised with surgery.

Case \#3 is a 11-year- old male with a primary mediastinal synovial sarcoma (SS) with part of the tumor mass infiltrating the superior vena cava and the upper part of the right atrium. The right pulmonary artery was also involved as well as the right main bronchi with no separation plane from the tumor.

2. The 3D models:

To create the 3D models a computerized aid designer (CAD) engineer, along with an expert pediatric radiologist and the leading surgeon, used CT images to delineate the tumor, the anatomical relations and the major vessels encased, working with tools that use color ranges (contrasting the vessels and bone mass) and manually (with the soft tissues) selecting image by image the differentiated parts. The software used for that purpose was VRMed DICOM Platform (10), developed in the ViRVIG research group (UPC University), and the resolution of the medical images was $1.5 \mathrm{~mm}$ of layer thickness in Case\#1 and Case\#2 and $0.3 \mathrm{~mm}$ in Case\#3

To ensure the right reproduction of the real anatomy, the pediatric radiologist and the surgeon, monitored all the work with review meetings and the team used MRI images to compare and confirm results.

The time spent on the 3D model generation was around 10 hours per case.

In each case, specific measures were taken in response to the acquired experience and the specific needs.

Case\#1: a 3D virtual model was obtained from routine abdomen CT and MRI images. When the virtual model was created (Figure 1a) it was again reviewed by the same radiologist and the oncologic surgeon prior to 3D printing. The objective was to create a model where the part representing the tumor had an "operable" translucent and soft texture that allowed visualization of the encased vessels and surrounding anatomical structures so that the surgical team could "operate" and "play" with it prior to the day of the actual surgery. The technology applied was Polyjet 3D Printing using a Connex 500 machine by Stratasys. Two different 3D files were created. One including bones, vessels and other parts was built using a white rigid opaque epoxy photopolymer. A different material was used to print the other file which reproduced the tumor that was soft and translucent (Figure 1b). Manufacturing a Polyjet 3D model can take around 24 hours.

Case \#2: the DICOM images already anticipated that the 3D printed tumor would not be able to show most of the encased vessels. The anatomical positioning of the vessels inside the 
tumor mass made difficult to accomplish the main goal of the surgical planning using this technology, which was to visualize before the real surgery the vessels potentially in danger from the tumor resection. Therefore, it was decided to complement the Polyjet 3D model with a second model made by SLS in a Vanguard machine by 3DSystems. It consisted of two parts, the first including the encased vessels with its anatomical relations; and the second including the tumor which could be removed so one could actually see the vessels and the rest of the anatomy without the tumor (Figure 2).

Case \#3: three prototypes were printed including the same two as in Case \#2 and a third one which represented the tumor alone in order for the surgical team to have the tumor volume "in hands" before the surgery (Figure 3). The third model was also made using SLS technology.

For Case \#2, a 3D customized support system was built so the model was placed in the same position as the one intended for the surgery (lateral decubitus). This improvement was printed with FFF open-source technology [11].

The models could be sterilized with Steam Formaldehyde at 60-80 드, thus allowing the models to be ready available for checking at any time during the surgical procedure.

\section{RESULTS:}

The models were completed one week before the planned surgery so the surgical team was able to study the case and operate on them well enough in advance. The prototypes were to real scale of the patient's organs thus giving an impression of what to expect during surgery. Their soft consistency, allowed the use of the different surgical instruments that would be used in the real surgery. The models gave surgeons a new tool for the surgical planning. This was especially of good use to residents, fellows and young surgeons. The fact that selected tumors were complicated and not very common, also gave the leading surgeon more confidence for the surgery. All patients underwent surgery as planned successfully. A right thoracoabdominal (TA) incision was used in Case \#1. Gross total resection (GTR) of the tumor encasing major vessels was achieved with no complications. The volume of the tumor correlated with the prototype (Figure 4). A TA incision was also used in Case \#2. GTR was performed without complications. For Case \#3, a medium sternotomy was performed. Cannulation of the heart and extracorporeal circulation was performed as planned. The tumor was totally removed along with a portion of the right atrium (RA) and superior vena cava (SVC), both invaded by the tumor. A tubularized dacron prosthesis was used to reconstruct the defect in the RA and SVC. The 3D models were used in real time during the surgeries to reassess the steps to be taken in order to remove the tumor mass preserving the encased vessels. All models predicted precisely the surgical findings in terms of tumor volume as well as vascular relationships.

\section{DISCUSSION:}


Rapid prototyping is the creation of a physical 3D model from computer design. The 3D printer uses the information of a virtual 3D model (obtained from a scanner or a 3D-drawing) to print the final structure [12][13]. Most of the reported clinical applications are in maxillofacial surgery, medical education, training, research, and lately, implant and tissue designing $[3,14]$. Several studies have demonstrated the efficacy of 3D models for the planning of maxillofacial surgeries $[15,16,17]$. A more accurate diagnosis and better understanding of complex anatomy as well as the possibility of pre-planning are key in the implementation of these technologies with better results [18]. Rapid prototyping also enhances quick learning and the possibility of case simulations $(19,20,21)$ highlighting important aspects in reducing risks during surgery and patient post-operative complications [9].

In this study we have explored new developments in technology and clinical feasibility of newly designed 3D printed models of complex soft tissue tumors with their anatomical relationships. By printing three different models of each tumor; the tumor and its relationships, the anatomy without the tumor and the tumor volume, we were able to explore different aspects required for detailed surgical planning. The integrated model allowed us to practice on the prototype and simulate the surgery before the operation. The consistency of the tumor material allowed us to dissect it with regular surgical instruments, cut it, and peel it away from organs and vessels. We also could practice different surgical approaches and weigh up the risks and benefits of each option in advance.

The printing of the tumor volume alone was also very useful. Some tumors are capsulated and well defined, but with others, such as the majority of high-risk neuroblastomas and sarcomas, tumor limits might be difficult to assess. So much so, that sometimes the removal of the tumor has to be done piecemeal. In those cases, having the tumor volume printed, definitely helps the surgeon to evaluate the grade of resection and objectively quantify a GTR.

The major technical drawback of the current models is that the vessels and organs have a rigid consistency. Despite the fact that their anatomical relations with the tumor are very accurate, at the time of surgical dissection they did not behave in the same elastic way as in the real anatomy. This is why having a prototype without the tumor was very useful so we could actually foresee the anatomy of the blood vessels embedded within the tumor.

This is a new technological development in an early stage with little data. Research is ongoing in our laboratories to improve the elasticity of the different densities of soft tissues.

Furthermore, since the cost of the models is a determinant factor in making surgical planning with 3D printed prototypes a standard for challenging surgical cases, further improvements in technology need to be developed. We are using open-source based technology and it is our belief that only open 3D-printing can face this challenge in the future. We welcome the surgical community to share its developments in order to improve to a more realistic model.

\section{ACKNOWLEDGEMENTS:}

The authors would like to thank; Margarita Vancells MD, JM Caffarena MD and Rosalia Carrasco MD PhD for their help in the work of this manuscript. 


\section{BIBLIOGRAPHY:}

1. Ventola CL. Medical Applications for 3D Printing: Current and Projected Uses. Pharmacy and Therapeutics. MediMedia, USA; 2014;39:704.

2. Webb PA. A Review of rapid prototyping (RP) techniques in the medical and biomedical sector. J Med Eng Technol. 2000;24:149-53.

3. Rengier F, Mehndiratta A, Tengg-Kobligk $H$, Zechmann $C M$, Unterhinninghofen $R$, Kauczor HU, et al. 3D printing based on imaging data: review of medical applications. Int J CARS. 2010;5:335-41.

4. Mavili ME1, Canter HI, Saglam-Aydinatay B, Kamaci S, Kocadereli I. Use of threedimensional medical modeling methods for precise planning of orthognathic surgery.J Craniofac Surg. 2007;18:740-7.

5. Mankovich NJ1, Cheeseman AM, Stoker NG. The display of three-dimensional anatomy with stereolithographic models.J Digit Imaging. 1990;3:200-3.

6. Ikegami T1, Maehara Y. Transplantation: 3D printing of the liver in living donor liver transplantation.Nat Rev Gastroenterol Hepatol. 2013;10:697-8.

7. Jacobs S, Grunert R, Mohr FW, Falk V. 3D-Imaging of cardiac structures using 3D heart models for planning in heart surgery: a preliminary study. Interact Cardiovasc Thorac Surg. 2008;7:6-9.

8. Costello JP, Olivieri $\mathrm{L}$, Krieger A, Thabit O, Marshall MB5, Yoo SJ, Kim PC, Jonas RA, Nath DS. Utilizing Three-Dimensional Printing Technology to Assess the Feasibility of HighFidelity Synthetic Ventricular Septal Defect Models for Simulation in Medical Education.World J Pediatr Congenit Heart Surg. 2014;5:421-426.

9. Silberstein JL, Maddox MM, Dorsey P, Feibus A, Thomas R, Lee BR. Physical models of renal malignancies using standard cross-sectional imaging and 3-dimensional printers: a pilot study. Urology. 2014;84:268-72.

10. Monclús Eva, Muñoz-Pandiella Imanol , Brunet Pere, Conesa Gerardo. Ventricular puncture trainer. CEIG 2012: XXII Spanish Conference of Computer Graphics. 2012; 45-48

11. Horne, Richard. From reprap students to masters of 3d printing - A Spanish success story. Disruptive Magazine, 2015 May 21. Available from http://disruptivemagazine.com/blog/fromreprap-students-to-masters-of-3d-printing-a-spanish-success-story-part-1/

12. Chua CK, Leong KF, LimCS. Rapid Prototyping Principles and applications .3rd Edition.Singapore: World Scientific, 2010.

13. Griffith ML, Harwell LD, Romero JT, Schlienger E, Atwood CL, Smugeresky JE. Multimaterial processing by LENS.Proceedings of the 1997 Solid Freeform Fabrication Symposium.1997;8:387-93 
14. Murphy SV, Atala A. 3D bioprinting of tissues and organs.Nat Biotechnol. 2014;32:773-

85.

15. Müller A, Krishnan KG, Uhl E, Mast G. The application of rapid prototyping techniques in cranial reconstruction and preoperative planning in neurosurgery. J Craniofac Surg. 2003;14:899-914.

16. D'Urso PS, Barker TM, Earwaker WJ, Bruce LJ, Atkinson RL, Lanigan MW, et al. Stereolithographic biomodelling in cranio-maxillofacial surgery: a prospective trial. J Craniomaxillofac Surg. 1999;27:30-7.

17. Esses SJ, Berman P, Bloom Al, Sosna J. Clinical applications of physical 3D models derived from MDCT data and created by rapid prototyping. AJR Am J Roentgenol. 2011;196:W683-8.

18. Mao K, Wang Y, Xiao S, Liu Z, Zhang Y, Zhang X, et al. Clinical application of computerdesigned polystyrene models in complex severe spinal deformities: a pilot study. Eur Spine J. 2010;19:797-802.

19. Schievano S, Migliavacca F, Coats L, Khambadkone S, Carminati M, Wilson N, et al. Percutaneous pulmonary valve implantation based on rapid prototyping of right ventricular outflow tract and pulmonary trunk from MR data. Radiology. 2007;242:490-7.

20. Nakada T, Akiba T, Inagaki T, Morikawa T. Thoracoscopic anatomical subsegmentectomy of the right S2b + S3 using a 3D printing model with rapid prototyping. Interact Cardiovasc Thorac Surg. 2014;19:696-8.

21. Sulaiman A, Boussel L, Taconnet F, Serfaty JM, Alsaid H, Attia C, Huet L, Douek P. In vitro non-rigid life-size model of aortic arch aneurysm for endovascular prosthesis assessment Eur J Cardiothorac Surg. 2008;33:53-7. 


\section{FIGURE CAPTIONS:}

\section{Table 1:}

Patients Characteristics

\section{Figure 1:}

a) 3D virtual reconstruction of case 1 tumor encassing major vessels from CT and MRI fusion images.

b) 3D printed prototype of case 1 . Tumor is represented in a semitransparent, "operable" consistency.

\section{Figure 2:}

a) $3 \mathrm{D}$ printed prototype of case 2 . Right mass crossing the midline with encasement of Celiac Trunk, SMA, IMA, Renal arteries, Renal veins, IVC, Portal Vein and hepatic duct. Tumor is also represented in a semitransparent, "operable" consistency.

b) Printed prototype of the same case with the tumor removed allowing the view of encased vessels.

\section{Figure 3:}

a) 3D printed prototype of mediastinal tumor with invasion of SVC and with no plane of separation with trachea, main right bronchus, right pulmonary vein and superior right lobule artery.

b) 3D printed prototype of tumor volume.

c) $3 \mathrm{D}$ printed prototype of the same case, tumor free.

\section{Figure 4:}

Case 1 removed tumor and prototype. Main tumor volume is the same as the 3D printed. Remaining tumor encasing major vessels was removed piecemeal. 